\title{
Oxidative Polymerization of N-Phenylanthranilic Acid in the Heterophase System
}

\author{
Sveta Zhiroslanovna Ozkan*, Igor Sergeevich Eremeev, \\ Galina Petrovna Karpacheva, Galina Nikolaevna Bondarenko \\ Russian Academy of Sciences, A. V. Topchiev Institute of Petrochemical Synthesis, Moscow, Russia \\ Email: "gpk@ips.ac.ru
}

Received April 1, 2013; revised April 30, 2013; accepted May 8, 2013

Copyright (C) 2013 Sveta Zhiroslanovna Ozkan et al. This is an open access article distributed under the Creative Commons Attribution License, which permits unrestricted use, distribution, and reproduction in any medium, provided the original work is properly cited.

\begin{abstract}
Polymers of N-phenylanthranilic acid were obtained by oxidative polymerization in the heterophase system in the presence of chloroform. Effect of synthesis conditions on the chemical structure of the polymers was studied. It was found that the growth of polymeric chain occurs via $\mathrm{C}-\mathrm{C}$ joining into 2- and 4-positions of phenyl rings with respect to nitrogen. Thermal stability of poly-N-phenylanthranilic acid was studied.
\end{abstract}

Keywords: Oxidative Polymerization; Poly-N-phenylanthranilic Acid; Chemical Structure

\section{Introduction}

Development of modern technologies demands new materials. Electroactive polymers attract large attention of researchers due to a complex of useful properties [1-5]. Polyaniline obtained via oxidative polymerization of aniline in aqueous solutions of acids is the most frequently used electroactive polymer nowadays [6,7]. Elaboration of new electroactive polymers is restrained by limited solubility of new monomers in aqueous solutions of acids. In recent years methods of interfacial oxidative polymerization of monomers which are not soluble in aqueous solutions of acids were developed. In case of interfacial polymerization, reagents (the monomer and the oxidant) are dispersed in two immiscible phases: the monomer is in the organic medium and the oxidant is in the aqueous medium; growth of the polymer proceeds at the interface [3,4,8-11]. Variation of organic solvents allows to involving monomers, which are not soluble in aqueous solutions of acids, into oxidative polymerization. The segregation of the oxidant into a separate phase gives an opportunity to change a type and concentration of the monomer without destroying the unstable oxidation products. Since the oxidation process occurs at the interface there is no need in gradual dispensing of reagents which is typical for polyaniline synthesis.

A serious problem of electroactive polymers is their

*Corresponding author. limited solubility in organic solvents. It is known that the introduction of carboxyl groups into the aromatic ring of polyaniline renders it soluble in aqueous alkaline solutions. Homopolymerization of anthranilic acid was studied in [12-14]. The reaction was conducted with ammonium and sodium persulfates both in the presence of sulfuric acid and without it. The yield of the polymer was not higher then $6 \%-8 \%$, and its electrical conductivity did not exceed $10^{-8} \mathrm{~S} / \mathrm{cm}$. The obtained polymer dissolves in aqueous solutions of $\mathrm{NH}_{4} \mathrm{OH}$ and $\mathrm{NaOH}$.

Copolymerization of anthranilic acid and aniline leads to the yield increase up to $40 \%-50 \%$. Percentage of links with carboxyl group reaches $60 \%$. However, growth of the amount of anthranilic acid in the reaction mixture causes MM of the copolymer to decrease from $M_{w}=1.3 \times 10^{5}$ to $8.4 \times 10^{4}$. Solubility improves as the number of anthranilic acid links in the copolymer increases. At the same time there is a significant decrease of electrical conductivity from $4.5 \times 10^{-5}$ for the equimolar ratio to $1 \times 10^{-8} \mathrm{~S} / \mathrm{cm}$ for 0.75 fold excess of anthranilic acid links $[12,13]$. The copolymer is electroactive as opposed to polyanthranilic acid [13].

Anthranilic acid is also able to copolymerize with diphenylamine. Content of anthranilic acid links in the copolymer increases with the increase of its concentration in the initial reaction mixture $[15,16]$.

Investigation of oxidative polymerization of diphenylamine-2-carboxylic acid in $5 \mathrm{M} \mathrm{H}_{2} \mathrm{SO}_{4}$ and in ammonium hydroxide solution showed that chemical structure 
of the polymer is dependent on $\mathrm{pH}$ of the reaction medium. It was found that the growth of the polymer chain occurs via $\mathrm{C}-\mathrm{C}$-joining into para-position of phenyl rings with respect to nitrogen, during the chemical oxidative polymerization of diphenylamine-2-carboxylic acid in the solution of sulfuric acid [5]. On the other hand, if diphenylamine-2-carboxylic acid is polymerized in ammonium hydroxide solution, the growth of polymer chain occurs via $\mathrm{C}-\mathrm{C}$-joining into 2- and 4-positions of phenyl rings with respect to nitrogen [17]. MM of polymers amounts to $M_{w}=1.9 \times 10^{4}$. The obtained polymers are soluble in aqueous alkaline solutions similarly to the case of polyaniline in which carboxyl groups are introduced in the aromatic ring $[12,13,15,16]$.

In the current paper experimental results of oxidative polymerization of N-phenylanthranilic acid in the heterophase system in the presence of an organic solvent (chloroform), are presented for the first time. The effect of synthesis conditions on the chemical structure, morphology and physicochemical properties of the polymers was studied.

\section{Experimental}

\subsection{Materials}

N-phenylanthranilic acid (of analytical grade), sulfuric acid (of the reagent grade), ammonia (of the reagent grade), chloroform (of the reagent grade), DMF (Acros Organics) and methyl alcohol ("JT Baker") were used as received. Ammonium persulfate (of analytical grade) was purified by recrystallization. Aqueous solutions of reagents were prepared using distilled water.

\subsection{Method of Poly-N-phenylanthranilic Acid Synthesis}

The oxidative polymerization of N-phenylanthranilic acid was performed via dissolving the required amount of monomer in chloroform and dissolving the oxidant (ammonium persulfate) and $\mathrm{NH}_{4} \mathrm{OH}$ in distilled water. The ratio of volumes of aqueous and organic phases was 1:1. The solutions of organic and aqueous phases were mixed immediately without gradual dispensing of re- agents. The intensive mixing process was carried out using the electric stirrer with top drive RW 16 Basic by "IKA Werke" in a narrow cylindrical round two-neck flask (to increase the efficiency of mixing) at $0^{\circ} \mathrm{C}$. The mixture was precipitated in a ten-fold excess of $2 \% \mathrm{H}_{2} \mathrm{SO}_{4}$ solution. The obtained product was filtered off and washed many times with distilled water to remove the residues of reagents. According to elemental analysis there is a residual amount of sulfate ions in the polymer after its precipitating in $2 \% \mathrm{H}_{2} \mathrm{SO}_{4}$ solution. Due to the fact that the resulting polymer dissolves in $\mathrm{NH}_{4} \mathrm{OH}$, it is impossible to carry out its neutralization. Low molecular weight oli- gomers and residues of sulfate ions were removed via extraction with methanol in Soxhlet apparatus for 1 day or via dialysis in distilled water for 20 days. Afterwards the product was dried under vacuum to a constant weight.

\subsection{Measurements}

$\mathrm{MM}$ of N-phenylanthranilic acid polymers was measured by GPC using "Water's $150^{\circ} \mathrm{C}$ ", equipped with speakers PLgel 5 um MIXED-C, using N-methylpyrrolidone as an eluent at $T=60^{\circ} \mathrm{C}$. Eluent's flow rate $-1 \mathrm{ml} / \mathrm{min}$. Volume of the injected sample-150 $\mathrm{ml}$. Calibration was performed on polystyrene. RI-detector was used. Accuracy of MM definition is $\sim 5 \%$.

IR spectra of poly-N-phenylanthranilic acid samples were recorded using "IFS $66 \mathrm{v}$ " FTIR spectrophotometer in the range $4000-400 \mathrm{~cm}^{-1}$. Samples were prepared as $\mathrm{KBr}$ pellets.

Electronic absorption spectra of poly-N-phenylanthranilic acid samples in DMF were registered by means of UV-1700 spectrophotometer "Shimadzu" in the range $190-1100 \mathrm{~nm}$.

${ }^{13} \mathrm{C}$ NMR-spectra of poly-N-phenylanthranilic acid were recorded using "Bruker MSL-300" spectrometer in solutions of deuterated DMSO.

Analysis of poly-N-phenylanthranilic acid samples by XPS was performed with the help of a two-chamber device XSAM-800 "Kratos Analytical Ltd". Characteristic band $\operatorname{Mg} K_{\alpha}(h v=1253.6 \mathrm{eV})$ was used as an exciting radiation.

X-ray studies were carried out at room temperature using X-ray diffractometer of "Rigaku" with Bragg- Brentano focusing on $\mathrm{Cr} K_{\alpha}$-radiation in a continuous mode.

Morphology of poly-N-phenylanthranilic acid powder was studied via scanning electron microscopy using electron microscope JSVU3 of JEOE (Japan). Electrically conductive carbon layer was previously spread on the surface of the sample via thermal vacuum graphite sputtering.

TEM images were obtained with Phillips EM-301 (acceleration voltage $60-80 \mathrm{kV}$ ).

BET surface area test of poly-N-phenylanthranilic acid was conducted on Micromeritics ASAP 2020 via capillary nitrogen condensation method at $77 \mathrm{~K}$ in the region of relative pressure $\left(\mathrm{P} / \mathrm{P}_{\mathrm{o}}\right)$ from 0.01 to 0.99 . Surface area correction factor is \pm 1 . Degassing of the sample was carried out at $120^{\circ} \mathrm{C}$ for 2 hours.

Thermal analysis was performed using TGA/DSC1 "Mettler Toledo" under dynamic heating in the temperature range $30^{\circ} \mathrm{C}-800^{\circ} \mathrm{C}$ in air and in nitrogen flow. The sample weight was $100 \mathrm{mg}$, the heating rate $-10^{\circ} \mathrm{C} / \mathrm{min}$, the nitrogen flow rate $-10 \mathrm{ml} / \mathrm{min}$. Calcined aluminium oxide was employed as a reference. The samples were analyzed in an $\mathrm{Al}_{2} \mathrm{O}_{3}$ crucible.

DSC analysis was carried out using DSC $823^{\mathrm{e}}$ "Mettler 
Toledo" calorimeter. Samples heating rate was $10^{\circ} \mathrm{C} / \mathrm{min}$ in argon atmosphere (its feeding rate was $70 \mathrm{ml} / \mathrm{min}$ ). Processing of experimental results was performed with the help of the service program STARe. The accuracy of measurements for the temperature determination was $\pm 0.3 \mathrm{~K}$ and for the enthalpy determination was $\pm 1 \mathrm{~J} / \mathrm{g}$.

\section{Results and Discussion}

\subsection{Synthesis of Poly-N-phenylanthranilic Acid}

The effect of concentration of monomer, oxidant, $\mathrm{NH}_{4} \mathrm{OH}$, temperature and time of synthesis on the yield and molecular-mass characteristics of poly-N-phenylanthranilic acid was studied. Analysis of the obtained data showed that high yield and maximum MM could be obtained under the following synthesis conditions: [monomer] $=0.1-0.2 \mathrm{~mol} / \mathrm{l} ;$ [oxidant] $=0.2-0.5 \mathrm{~mol} / \mathrm{l}$; $\left[\mathrm{NH}_{4} \mathrm{OH}\right]=0.5-1.0 \mathrm{~mol} / \mathrm{l} ; T=0^{\circ} \mathrm{C}-15^{\circ} \mathrm{C} ; t=3-6$ hours. Maximum yield of the polymer was $72 \%-79 \%$. According to GPC, molecular mass of the polymer reached $M_{w}=2.6 \times 10^{4}$, the degree of polymerization was more than 120 , polydispersity index was 2.2 .

$\mathrm{N}$-phenylanthranilic acid polymer is a black powder, completely soluble in aqueous solutions of $\mathrm{NH}_{4} \mathrm{OH}$ and $\mathrm{NaOH}$, N-methylpyrrolidone, DMF, DMSO, partially in THF, dioxane, acetone. According to X-ray and TEM analyses poly-N-phenylanthranilic acid is an amorphous polymer (Figures 1 and 2).

\subsection{Chemical Structure of Poly-N-phenylanthranilic Acid}

Structure of N-phenylanthranilic acid polymers was studied by FTIR and NMR spectroscopy and XPS.

Figure 3 shows a comparison of the IR spectra of the monomer (a) and the obtained polymer (b). In the IR spectrum of poly-N-phenylanthranilic acid there are intensive bands at 1590 and $1510 \mathrm{~cm}^{-1}$, which refer to the stretching vibrations of the $v_{\mathrm{C}-\mathrm{C}}$ bonds in aromatic rings. Splitting of these bands indicates the different type of the substitution of aromatic rings. Bands at 1683 and 1227 $\mathrm{cm}^{-1}$ refer to the vibrations of $\mathrm{COOH}$ groups. In the monomer these bands are observed at 1658 and 1259 $\mathrm{cm}^{-1}$. Absorption band at $1309 \mathrm{~cm}^{-1}$ refers to the stretching vibrations of $v_{\mathrm{C}-\mathrm{N}}$ bonds $[5,17]$.

The presence of the bands of monosubstituted phenyl rings (nonplanar deformation vibrations of bonds $\delta_{\mathrm{C}-\mathrm{H}}=$ $746,697 \mathrm{~cm}^{-1}$ ) is caused by the end groups [5,17].

Broadening and shift to $750 \mathrm{~cm}^{-1}$ of the band at 746 $\mathrm{cm}^{-1}$ and presence of a broad band at $830 \mathrm{~cm}^{-1}$ in the IR spectrum of poly-N-phenylanthranilic acid refer to the presence of 1,2-disubstituted and 1,2,4-threesubstituted aromatic rings in the polymer structure. It shows that the growth of the polymer chain proceeds via $\mathrm{C}-\mathrm{C}$ - joining in 2- and 4-positions of phenyl rings with respect to ni-

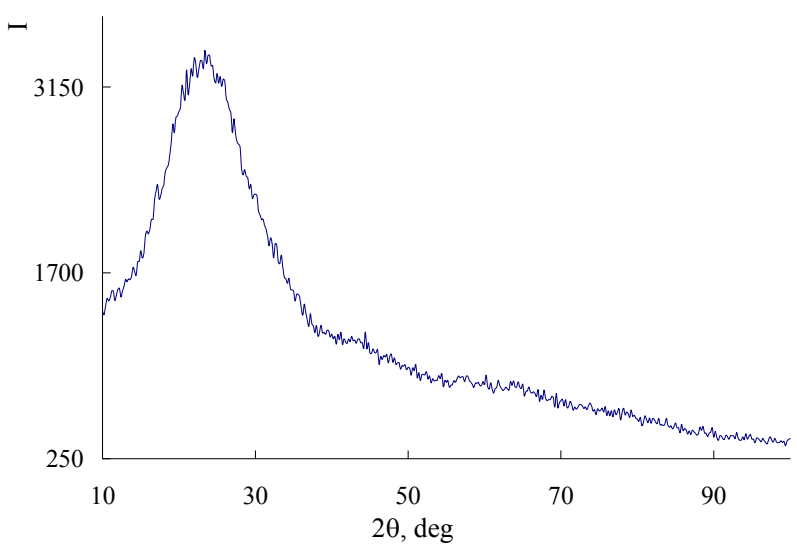

Figure 1. XRD spectrum of poly-N-phenylanthranilic acid.

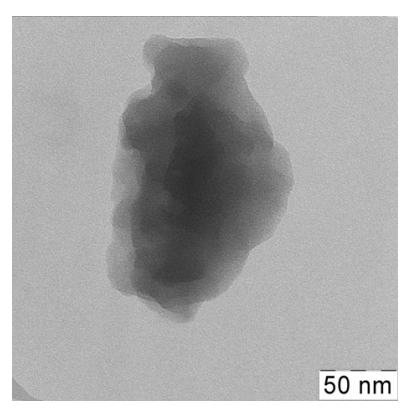

(a)

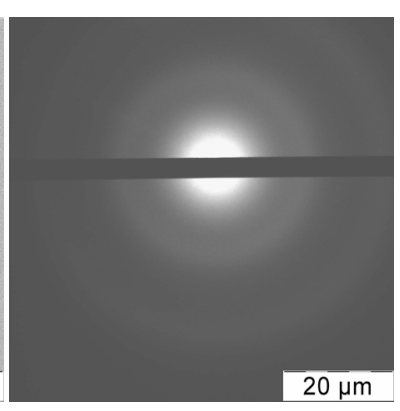

(b)
Figure 2. TEM image (a) and diffraction (b) of poly-Nphenylanthranilic acid.

trogen as it takes place in the case of polymerization of $\mathrm{N}$-phenylanthranilic acid in the solution of ammonium hydroxide [17].

In the IR spectrum of the monomer the band of the stretching vibrations of $v_{\mathrm{C}=\mathrm{O}}$ bonds in the carboxyl group appears at $1658 \mathrm{~cm}^{-1}$. It means that it is strongly shifted to longer wavelengths compared to the normal position of these bands $\left(1730-1710 \mathrm{~cm}^{-1}\right)$ [5]. Such a shift of $v_{\mathrm{C}=\mathrm{O}}$ band together with the appearance of a number of weak bands in the range of $2490-2640 \mathrm{~cm}^{-1}$ indicate that when the monomer is in a solid phase there is dimerization at the carboxyl group with a formation of a dimer structure:<smiles>CC1=COC(C)OOC(C)O1</smiles>

In this case the absorption band of stretching vibrations of $\nu_{\mathrm{N}-\mathrm{H}}$ bonds becomes visible near $3337 \mathrm{~cm}^{-1}$, where bands of non-associated aminogroups are usually located. In the IR spectrum of the polymer $v_{\mathrm{C}=\mathrm{O}}$ band shifts to shorter wavelengths to $1683 \mathrm{~cm}^{-1}$, while band of the $\mathrm{N}-\mathrm{H}$ bond strongly broadens and shifts to longer wavelengths $\left(3239 \mathrm{~cm}^{-1}\right)$, and broad bands from the dimer of carboxyl group in the range of $2490-2640 \mathrm{~cm}^{-1}$ almost disappear. The above mentioned changes are spectral features of the fact that dimers of carboxyl 


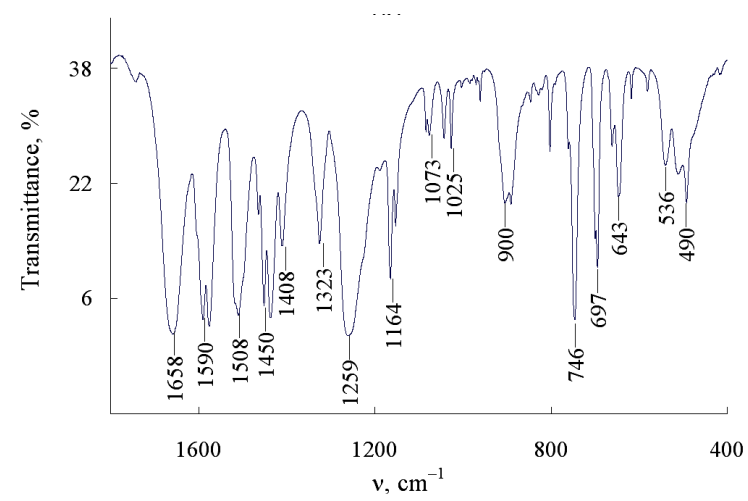

(a)

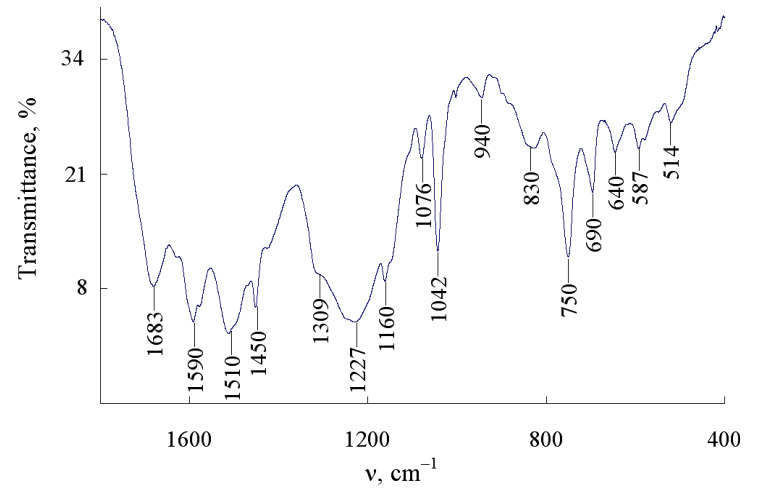

(b)

Figure 3. (a) FTIR spectra of the monomer and (b) poly-Nphenylanthranilic acid. [Monomer] $=0.1$, [oxidant] $=0.2$, $\left[\mathrm{NH}_{4} \mathrm{OH}\right]=0.5 \mathrm{~mol} / \mathrm{l}, \mathrm{T}=0^{\circ} \mathrm{C}$, synthesis time $3 \mathrm{~h}$.

groups are destroyed during polymerization. $\mathrm{COOH}$ groups associate with $\mathrm{N}-\mathrm{H}$ group of the main polymer chain. Carboxyl groups along the whole polymer chain form intramolecular hydrogen bonds with amino groups, which is proved by the appearance of a band near 3288 $\mathrm{cm}^{-1}$ in the IR spectrum and a band $\lambda_{\max }=550 \mathrm{~nm}$ in the electronic absorption spectrum of the polymer (Figure 4). This band is absent in the electronic absorption spectrum of the monomer.

Analysis of the results of spectral studies allows to representing a chemical structure of poly-N-phenylanthranilic acid, obtained via oxidative polymerization in the presence of chloroform, as follows:

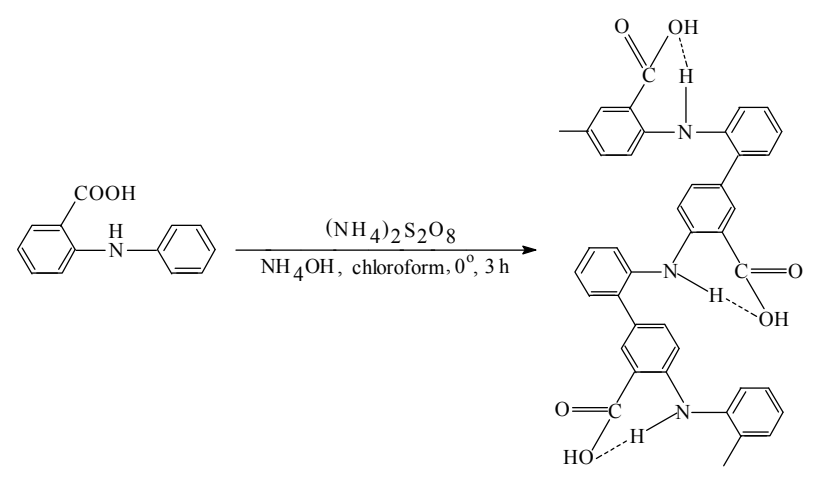

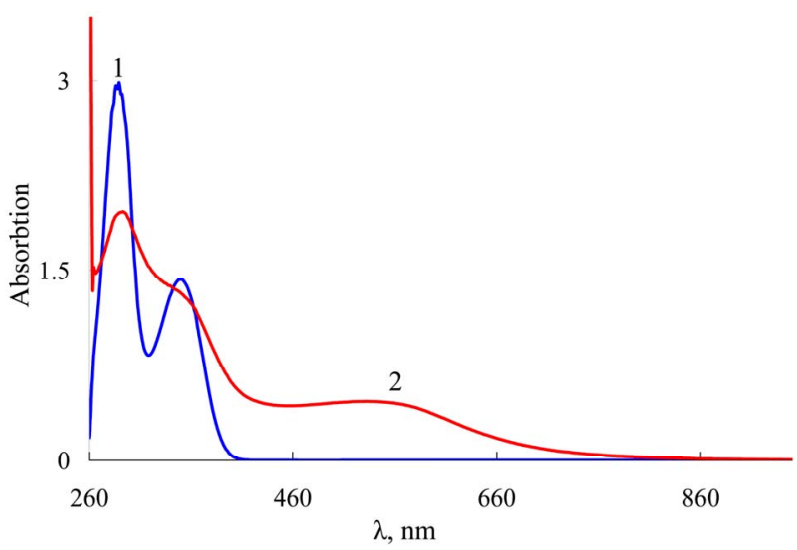

Figure 4. Electronic absorption spectra of the monomer (1) and poly-N-phenylanthranilic acid in DMF (2). [Monomer] $=0.1$, $[$ oxidant $]=0.2,\left[\mathrm{NH}_{4} \mathrm{OH}\right]=0.5 \mathrm{~mol} / \mathrm{l}, \mathrm{T}=0^{\circ} \mathrm{C}$, synthesis time $3 \mathrm{~h}$.

The effect of oxidant concentration on the structure of poly-N-phenylanthranilic acid was studied. It was found that as the ratio [oxidant]:[monomer] grows in a series $1.25,2.0,5.0$, the intensity of bands of monosubstituted phenyl rings decreases $\delta_{\mathrm{C}-\mathrm{H}}=746,697 \mathrm{~cm}^{-1}$ (intensity of bands was measured by the base line). It means that polymerization degree increases. GPC data prove that polymerization degree increases with the growth of concentration of the oxidant in the reaction medium.

An important feature of poly-N-phenylanthranilic acid structure is an absence of quinodiimine units, which is evidenced by XPS, NMR and electronic spectroscopy. In the structure of the polymer there are only phenyleneamine units. In the $\mathrm{N} 1 \mathrm{~s}$ spectrum of poly-N-phenylanthranilic acid there is no band near $399.0 \mathrm{eV}$, which corresponds to the energy of $\mathrm{C}=\mathrm{N}$ bond. The band observed near $400.5 \mathrm{eV}$ refers to the energy of $\mathrm{C}-\mathrm{N}$ bond $[5,18$ 21].

Electronic spectra confirm these observations. It was shown that there is no absorption band in the long wavelength range, which originates from oxidized quinoid form, even in cases of excess of stoichiometric molar ratio oxidant: monomer. For example, for polyaniline, this band is situated in the region of $\lambda_{\max }=620 \mathrm{~nm}$, for polyphenothiazine in the region of $\lambda_{\max }=640 \mathrm{~nm}$ [10]. The absence of quinodiimine units in the structure of polydiphenyamine-2-carboxylic acid is also confirmed by ${ }^{13} \mathrm{C}$ NMR data. In the ${ }^{13} \mathrm{C}$ NMR spectrum of the polymer there is no signal which corresponds to the carbon atoms from $\mathrm{C}=\mathrm{N}$ groups with a chemical shift $\delta_{\mathrm{C}}=148$ ppm [10].

\subsection{Morphology}

When the system is intensively stirred, the dispersive reaction medium is formed. The continuous phase is the 
aqueous alkaline solution with an oxidant and the dispersive phase consists of the drops of monomer solution in chloroform. The significant difference of the process in view from the interfacial one, is the fact that only the initiation of the polymerization proceeds on the interface. Since the monomer dissolves not only in chloroform but also in the aqueous alkaline solution, the growth of the polymer chain takes place in the aqueous medium, with a gradual transfer of the monomer from the organic phase into aqueous. As a result, on the place of the chloroform drops cavities are formed, which is proved by SEM (Figure 5). The polymer synthesized during N-phenylanthranilic acid polymerization in the $\mathrm{NH}_{4} \mathrm{OH}$ solution has a plate-like morphology. Surface area was estimated by BET surface area test. It was equal to $44 \mathrm{~m}^{2} / \mathrm{g}$. Pore volume: $0.113 \mathrm{~cm}^{3} / \mathrm{g}$, average pore size: $10 \mathrm{~nm}$. The total porosity is not large and is $9 \%$.

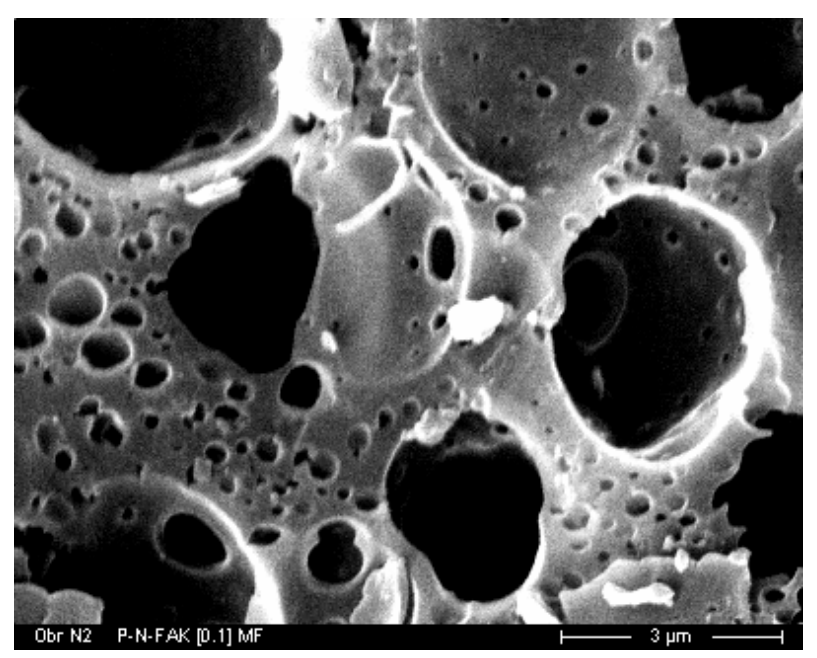

(a)

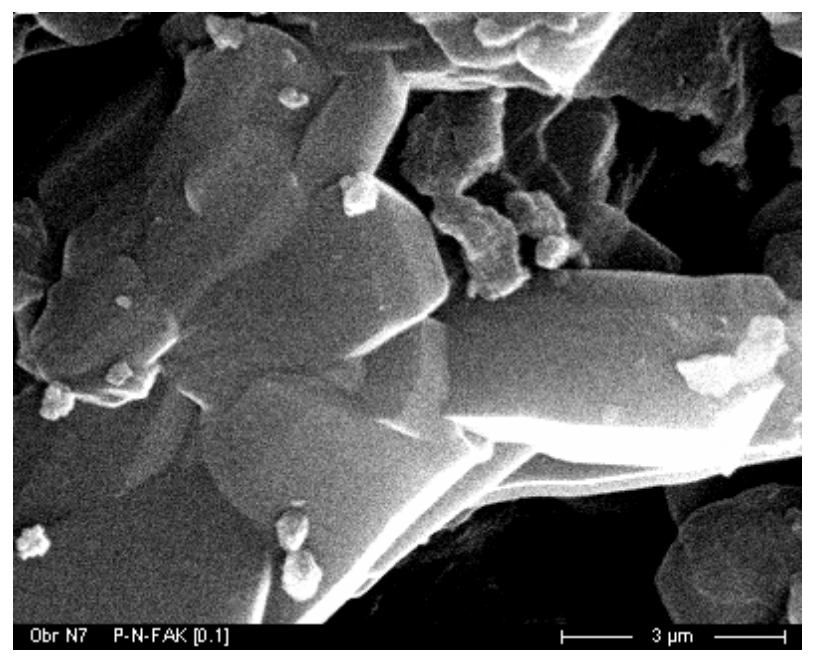

(b)

Figure 5. SEM images of poly-N-phenylanthranilic acid, obtained by polymerization in the presence of chloroform (a) and in $\mathrm{NH}_{4} \mathrm{OH}$ solution (b).

\subsection{Thermal Stability of Poly-N-phenylanthranilic Acid}

Thermal stability of poly-N-phenylanthranilic acid was studied via methods of TGA and DSC. Figure 6 shows temperature dependence of weight loss of poly-Nphenylanthranilic acid heated to $800^{\circ} \mathrm{C}$ in nitrogen flow and in air. The curves of weight loss take a step-like shape. Weight loss at low temperatures $\left(\sim 90^{\circ} \mathrm{C}\right)$ in poly$\mathrm{N}$-phenylanthranilic acid is caused by the removal of moisture [22-25]. It is also confirmed by the DSC data (Figure 7). Weight loss at $168^{\circ} \mathrm{C}$ is associated with the removal of $\mathrm{COOH}$ groups $[5,14]$. The DSC curves of poly-N-phenylanthranilic acid in this temperature range show an exothermic peak indicating the decomposition [26-28]. This peak is absent after reheating. Poly-Nphenylanthranilic acid loses half of its weight in air at $520^{\circ} \mathrm{C}$. In the inert atmosphere $50 \%$ weight loss is observed at $660^{\circ} \mathrm{C}$. At $800^{\circ} \mathrm{C}$ the residue is $31 \%$.

\section{Conclusions}

The chemical oxidative polymerization of N-phenylan-

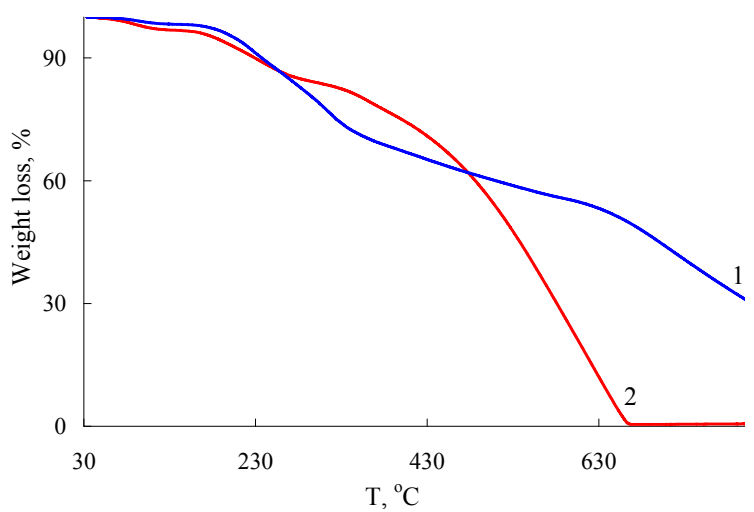

Figure 6. Weight loss of poly-N-phenylanthranilic acid while heating up to $800^{\circ} \mathrm{C}$ at rate $10^{\circ} \mathrm{C} / \mathrm{min}$ in nitrogen flow (1) and in air (2). [Monomer] $=0.1$, [oxidant] $=0.2,\left[\mathrm{NH}_{4} \mathrm{OH}\right]$ $=0.5 \mathrm{~mol} / \mathrm{l}, \mathrm{T}=0^{\circ} \mathrm{C}$, synthesis time $3 \mathrm{~h}$.

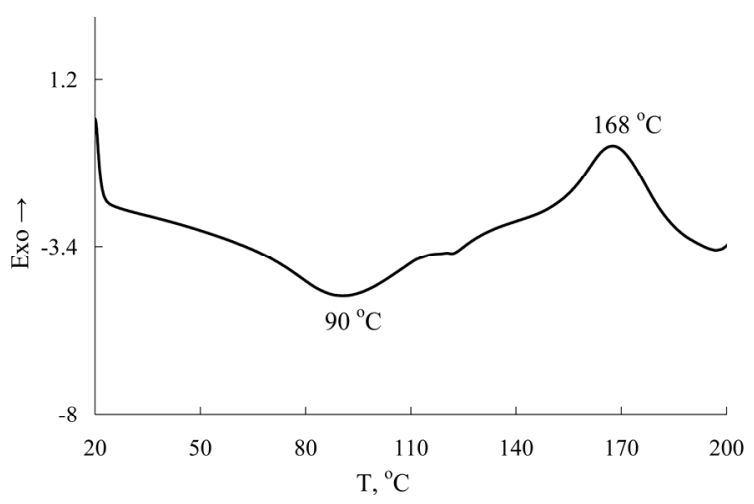

Figure 7. DSC thermogram of poly-N-phenylanthranilic acid while heating in nitrogen flow up to $200^{\circ} \mathrm{C}$ at rate $10^{\circ} \mathrm{C} /$ min. 
thranilic acid in the heterophase system was performed for the first time and the polyacid was obtained. In its structure carboxyl groups form intramolecular hydrogen bonds with $\mathrm{N}-\mathrm{H}$ groups along the whole polymer chain. IR spectroscopy showed that the growth of polymer chain occurs via C-C - joining into 2- and 4-positions of phenyl rings with respect to nitrogen. It was demonstrated that in spite of the excess of the oxidant in the structure of poly-N-phenylanthranilic acid there are only phenyleneamine units.

Heterocyclic polymers of N-phenylanthranilic acid are amorphous and have a high thermal stability. It was found that for poly-N-phenylanthranilic acid 50\% weight loss is observed at $660^{\circ} \mathrm{C}$ in inert atmosphere and $520^{\circ} \mathrm{C}$ in air. In nitrogen flow at $800^{\circ} \mathrm{C}$ the residue is $31 \%$.

\section{Acknowledgements}

The authors acknowledge the financial support from the Russian Foundation for Basic Research, project 11-0300560a. The authors thank T. N. Prudskova (Open Joint Stock Company "G. S. Petrov Institute of Plastics", Russia) for the determination of molecular-mass charac- teristics, N. A. Zhilyaeva (A. V. Topchiev Institute of Petrochemical Synthesis RAS) for the determination of polymers surface area and G. A. Shandryuk (A. V. Topchiev Institute of Petrochemical Synthesis RAS) for conducting of thermal analysis of polymers.

\section{REFERENCES}

[1] A. Malinauskas, "Chemical Deposition of Conducting Polymers," Polymer, Vol. 42, No. 9, 2001, pp. 39573972. doi:10.1016/S0032-3861(00)00800-4

[2] A. G. MacDiarmid, "Synthetic Metals: A Novel Role for Organic Polymers," Synthetic Metals, Vol. 125, No. 1, 2002, pp. 11-22. doi:10.1016/S0379-6779(01)00508-2

[3] Zh. Ozkan, G. P. Karpacheva, A. V. Orlov and M. A. Dzyubina, "Thermal Stability of Diphenylamine Synthesized through Oxidative Polymerization of Diphenylamine," Journal of Polymer Science B, Vol. 49, No. 1-2, 2007, pp. 36-41.

[4] S. Zh. Ozkan, G. P. Karpacheva and G. N. Bondarenko, "Polymers of Phenoxazine: Synthesis, Structure," Russian Chemical Bulletin, Vol. 60, No. 8, 2011, pp. 1651-1656. doi:10.1007/s11172-011-0247-z

[5] S. Zh. Ozkan, G. N. Bondarenko and G. P. Karpacheva, "Oxidative Polymerization of Diphenylamine-2-carboxylic Acid: Synthesis, Structure, and Properties of Polymers," Journal of Polymer Science B, Vol. 52, No. 5, 2010, pp. 263-269.

[6] P. N. Adams and A. P. Monkman, "Characterization of High Molecular Weight Polyaniline Synthesized at -40 ${ }^{\circ} \mathrm{C}$ Using a $0.25: 1$ Mole Ratio of Persulfate Oxidant to Aniline," Synthetic Metals, Vol. 87, No. 2, 1997, pp. 165169. doi:10.1016/S0379-6779(97)03818-6

[7] J. Stejskal, A. Riede, D. Hlavata, J. Prokees, M. Helms- tedt and P. Holler, "The Effect of Polymerization Temperature on Molecular Weight, Crystallinity, and Electrical Conductivity of Polyaniline," Synthetic Metals, Vol. 96, No. 1, 1998, pp. 55-61. doi:10.1016/S0379-6779(98)00064-2

[8] A. V. Orlov, S. Zh. Ozkan, G. N. Bondarenko and G. P. Karpacheva, "Oxidative Polymerization of Diphenylamine: Synthesis and Structure of Polymers," Journal of Polymer Science B, Vol. 48, No. 1-2, 2006, pp. 5-10.

[9] A. V. Orlov, S. Zh. Ozkan and G. P. Karpacheva, "Oxidative Polymerization of Diphenylamine: A Mechanistic Study," Journal of Polymer Science B, Vol. 48, No. 1-2, 2006, pp. 11-17.

[10] S. Zh. Ozkan, G. N. Bondarenko, A. V. Orlov and G. P. Karpacheva, "Interfacial Oxidative Polymerization of Phenothiazin," Journal of Polymer Science B, Vol. 51, No. 5-6, 2009, pp. 149-156.

[11] Y. M. Korolev and S. Zh. Ozkan, "Synthesis and X-Ray Diffraction Study of Polyphenothiazine," Doklady Physical Chemistry, Vol. 429, No. 1, 2009, pp. 223-226. doi:10.1134/S0012501609110025

[12] H. S. O. Chan, S. C. Ng, W. S. Sim, K. L. Tan and B. T. G. Tan, "Preparation and Characterization of Electrically Conducting Copolymers of Aniline and Anthranilic Acid: Evidence for Self-Doping by X-ray Photoelectron spectroscopy," Macromolecules, Vol. 25, No. 22, 1992, pp. 6029-6034. doi:10.1021/ma00048a026

[13] M. T. Nguen and A. F. Diaz, "Water-Soluble Poly(Aniline-co-o-Anthranilic Acid) Copolymers," Macromolecules, Vol. 28, No. 9, 1995, pp. 3411-3415. doi:10.1021/ma00113a047

[14] K. Ogura, H. Shiigi, M. Nakayama and A. Ogawa, "Thermal Properties of Poly(Anthranilic Acid) (PANA) and Humidity-Sensitive Composites Derived from HeatTreated PANA and Poly(Vinyl Alcohol)," Journal of Polymer Science Part A: Polymer Chemistry, Vol. 37, No. 23, 1999, pp. 4458-4465.

doi:10.1002/(SICI)1099-0518(19991201)37:23<4458::AI D-POLA23>3.0.CO;2-R

[15] M. S. Wu, T. C. Wen and A. Gopalan, "In Situ UV-Visisible Spectroelectrochemical Studies on the Copolymerization of Diphenylamine with Anthranilic Acid," Materials Chemistry and Physics, Vol. 74, No. 1, 2002, pp. 58-65. doi:10.1016/S0254-0584(01)00406-0

[16] M. S. Wu, T. C. Wen and A. Gopalan, "Electrochemical Copolymerization of Diphenylamine and Anthranilic Acid with Various Feed Ratios," Journal of The Electrochemical Society, Vol. 148, No. 5, 2001, pp. D65-D73. doi:10.1149/1.1366625

[17] S. Zh. Ozkan, I. S. Eremeev, G. P. Karpacheva, T. N. Prudskova, E. V. Veselova, G. N. Bondarenko and G. A. Shandryuk, "Polymers of Dipheylamine-2-carboxylic Acid: Synthesis, Structure and Properties," Journal of Polymer Science B, Vol. 55, No. 3-4, 2013, pp. 107-115.

[18] S. Zh. Ozkan, "Candidates Dissertation in Chemistry," 2006.

[19] A. P. Dementjev, A. de Graaf, M. C. M. van de Sanden, K. I. Maslakov, A. V. Naumkin and A. A. Serov, "X-Ray Photoelectron Spectroscopy Reference Data for Identification of the $\mathrm{C}_{3} \mathrm{~N}_{4}$ Phase in Carbon-Nitrogen Films," 
Diamond and Related Materials, Vol. 9, No. 11, 2000, pp. 1904-1907. doi:10.1016/S0925-9635(00)00345-9

[20] K. L. Tan, B. T. G. Tan, E. T. Kang and K. G. Neoh, "XRay Photoelectron Spectroscopy Studies of the Chemical Structure of Polyaniline," Physical Review B, Vol. 39, No. 11, 1989, pp. 8070-8073. doi:10.1103/PhysRevB.39.8070

[21] S. W. Huang, K. G. Neoh, E. T. Kang, H. S. Han and K. L. Tan, "Palladium-Containing Polyaniline and Polypyrrole Microparticles," Journal of Materials Chemistry, Vol. 8, No. 8, 1998, pp. 1743-1748. doi:10.1039/a802245c

[22] J. Yue, A. J. Epstein, Z. Zhong, P. K. Gallagher and A. G. MacDiarmid, "Thermal Stabilites of Polyanilines," Synthetic Metals, Vol. 41, No. 1-2, 1991, pp. 765-768. doi:10.1016/0379-6779(91)91180-I

[23] V. G. Kulkarni, L. D. Campbell and W. R. Mathew, "Thermal Stability of Polyaniline," Synthetic Metals, Vol. 30, No. 3, 1989, pp. 321-325. doi:10.1016/0379-6779(89)90654-1

[24] A. Boyle, J. F. Penneau, E. Genies and C. Riekel, "The Effect of Heating on Polyaniline Powders Studied by
Rreal-Time Synchrotron Radiation Diffraction, Mass Spectrometry and Thermal Analysis," Journal of Polymer Science Part B: Polymer Physics, Vol. 30, No. 1, 1992, pp. 265-274. doi:10.1002/polb.1992.090300306

[25] K. Amano, H. Ishikawa, A. Kobayashi, M. Satoh and E. Hasegawa, "Thermal Stability of Chemically Synthesized Polyaniline," Synthetic Metals, Vol. 62, No. 3, 1994, pp. 229-232. doi:10.1016/0379-6779(94)90210-0

[26] L. Ding, X. Wang and R. V. Gregory, "Thermal Properties of Chemically Synthesized Polyaniline (EB) Powder," Synthetic Metals, Vol. 104, No. 2, 1999, pp. 73-78. doi:10.1016/S0379-6779(99)00035-1

[27] X.-H. Wang, Y.-H. Geng, L.-X. Wang, X.-B. Jing and F.-S. Wang, "Thermal Behaviors of Doped Polyaniline," Synthetic Metals, Vol. 69, No. 1-3, 1995, pp. 265-266. doi:10.1016/0379-6779(94)02443-3

[28] T.-Ch. Wen, J.-B. Chen and A. Gopalan, "Soluble and Methane Sulfonic Acid Doped Poly(Diphenylamine)-Synthesis and Characterization," Materials Letters, Vol. 57, No. 2, 2002, pp. 280-290. 